Tropical Journal of Pharmaceutical Research March 2017; 16 (3): 649-656

ISSN: $1596-5996$ (print); 1596-9827 (electronic)

(C) Pharmacotherapy Group, Faculty of Pharmacy, University of Benin, Benin City, 300001 Nigeria.

All rights reserved.

Available online at http://www.tjpr.org

Original Research Article

http://dx.doi.org/10.4314/tjpr.v16i3.21

\title{
Protective effects of Naringin in a rat model of spinal cord ischemia-reperfusion injury
}

\author{
Yongsheng Cui*, Wei Zhao, Xinhua Wang, Mingxin Chen, Li Zhang, Zhilei Liu \\ Department of Orthopaedics II, Ankang City Central Hospital, Ankang, Shanxi 725000, China
}

*For correspondence: Email: yongshengcui28@hotmail.com; Tel/Fax: 0086-13-084813344

Received: 31 October 2016

Revised accepted: 12 February 2017

\begin{abstract}
Purpose: To evaluate the activity of naringin (NAR) in a rat model of spinal cord ischemic injury (SCII). Methods: Forty female rats were randomized into four groups: saline without occlusion (control; group I), SCII (group II), $50 \mathrm{mg} / \mathrm{kg}$ NAR (group III), or $100 \mathrm{mg} / \mathrm{kg}$ NAR (group IV) for 7 days prior to SCl insult (pre-treatment). Neurological and locomotor functions, antioxidant activity, edema and inflammatory markers were determined.

Results: Pre-treatment with NAR considerably lowered the incidence of spinal edema, lipid peroxidation products, and inflammatory markers (TNF- $\alpha, N F-p 65, I L-1 \beta$, and IL-6). It also successfully reverted the antioxidative activity to near-normal levels and improved locomotor function by protecting spinal tissue from oxidative damage and inflammatory insults. NAR administration effectively downregulated the protein expression of TNF- $\alpha$ and NF-KB p65 subunit in spinal tissue, thus confirming its antiinflammatory activity.

Conclusion: The results suggests that NAR exhibits neuroprotective effects by inhibiting free radical generation and downregulating inflammatory markers in an SCl rat model.
\end{abstract}

Keywords: Naringin, Spinal cord injury, Locomotor function, Edema, Oxidative stress, Inflammation

Tropical Journal of Pharmaceutical Research is indexed by Science Citation Index (SciSearch), Scopus, International Pharmaceutical Abstract, Chemical Abstracts, Embase, Index Copernicus, EBSCO, African Index Medicus, JournalSeek, Journal Citation Reports/Science Edition, Directory of Open Access Journals (DOAJ), African Journal Online, Bioline International, Open-J-Gate and Pharmacy Abstracts

\section{INTRODUCTION}

Spinal cord ischemic injury (SCII) is a serious condition that occurs after thoracoabdominal aortic aneurysm (TAAA) surgery and results in muscular disability, particularly paraplegia [1]. The incidence of paraplegia is $\sim 10-30 \%$ after thoracic aortic reconstruction. However, this incidence has increased enormously in recent times, making SCII a serious problem globally [2]. Many current treatment strategies such as hypothermia, partial bypass therapies, and pharmaceutical intervention are recommended to reduce the prevalence of SCII [3]. However, the occurrence of SCII remains high, and these treatments (synthetic drugs) and procedures may trigger various adverse events and thus worsen the patient health status. Hence, treating SCII is a major goal for health professionals and researchers to reduce the prevalence of paraplegia while avoiding adverse events [2].

Several pathological factors may contribute to SCII, but the exact mechanism remains unknown $[4,5]$. Numerous studies have suggested that oxidative stress (an imbalance between antioxidative and pro-oxidative factors) and inflammation are the pivotal events in SCII [3]. Hence, treating with phytocomponents that exhibit antioxidant and anti-inflammatory properties might alleviate SCll-related effects. Several recent studies also demonstrated that phytoconstituents such as polyphenols (phenolic or flavonoids) could effectively lower spinal 
edema, inflammation, and oxidative stress and improve neuronal activity (motor function) by inhibiting apoptosis $[6,7]$.

Naringin (NAR) is a bioflavanone glycoside that is found in citrus species. Although NAR cannot be absorbed directly, it can be metabolized by intestinal microflora to yield a metabolite called naringenin, which is an absorbable form of NAR that can effectively cross the blood-spinal cord barrier [8]. NAR exerts several therapeutic and pharmacological activities including antioxidative, anti-inflammatory, anti-angiogenic, antidiabetic, anticancer, and antibacterial effects $[9,10]$. NAR can also act as a neuroprotective agent against Alzheimer's disease (AD) and Parkinson's disease (PD). Moreover, NAR exhibits cardioprotective, hepatoprotective, and renoprotective properties $[11,12]$.

Furthermore, numerous pharmaceutical and nutritional companies have focused on NAR because of its antioxidative and antiinflammatory properties, and have started to manufacture it as a nutritive or dietary supplement [13]. NAR has also exhibited positive effects in organs such as the brain, heart, and kidney in experimental ischemic-reperfusion (IR) models by reducing oxidative stress and inflammation [14-16]. Nevertheless, no experiments have been conducted to investigate the effects of NAR in SCII, specifically regarding oxidative stress and inflammatory markers.

Hence, we speculate that NAR might protect against spinal cord ischemic injury by suppressing oxidative stress and the inflammatory response in an IR animal model without any adverse events.

\section{EXPERIMENTAL}

\section{Chemicals and reagents}

NAR, Tween 20, malondialdehyde (MDA), sodium dodecyl sulfate (SDS), Tris buffer, bromophenol blue, and bovine serum albumin (BSA) were purchased from Sigma-Aldrich (St. Louis, MO, USA). Ketamine, phosphate-buffered saline (PBS), and Tris-buffered saline (TBS), were obtained from Sangon Biotechnology (Shanghai, China). Superoxide dismutase (SOD), glutathione contents (GSH), catalase (CAT), myeloperoxidase (MPO), and lipid peroxidation product (MDA) commercial kits were purchased from the Nanjing Jiancheng Bioengineering Institute (Nanjing, China). All other chemicals used in this study were of analytic grade.

\section{Experimental animals}

Adult female Sprague-Dawley (SD) rats weighing $300-320 \mathrm{~g}$ were used in all experiments. They were housed in the animal facility maintained at $25-28{ }^{\circ} \mathrm{C}$ with $12 \mathrm{~h}$ light/dark cycles and free access to water and standard rat chow. All experimental procedures were approved by the ethics review board of Ankang City Central Hospital (no. ACCH-35630) and were in conformity with the revised guidelines of the US National Institutes of Health (NIH).

\section{SCll induction}

$\mathrm{SCl}$ injury/insult was performed using the Hwang method [17] with a slight modification. All rats were anesthetized by intraperitoneal (i.p.) injection of $40 \mathrm{mg} / \mathrm{kg}$ ketamine and maintained at $37^{\circ} \mathrm{C}$ with heating pads and an infrared lamp. A polyethylene catheter was inserted into the tail artery to facilitate continuous monitoring of mean arterial pressure (MAP) and to check the heart rate. A Fogarty balloon catheter was inserted into the descending thoracic aorta through the left femoral artery (midline insertion) and then inflated to induce spinal cord ischemia (occlusion). The ischemia was confirmed by a sudden drop in MAP and heart rate. Then, after 30 min of occlusion, reperfusion (restored blood flow) was initiated by deflating the balloon. Finally, the Fogarty balloon catheter was carefully removed from thoracic aorta, and the rats were allowed to recover from anesthesia. For control rats, all the procedures were carried out except aortic occlusion.

\section{Experimental groups}

Forty healthy female SD rats were randomly chosen and separated into four groups of 10 rats ( $n=10$ /group). Group I rats received only saline without occlusion (sham-operated control). Group II rats underwent SCII, whereas Group III and IV rats underwent SCII and were treated with 50 and $100 \mathrm{mg} / \mathrm{kg}$ NAR (dissolved in saline), respectively, via i.p. injection for 7 days before receiving the SCII insult (pre-treatment) and thus served as the treatment groups (Group III, NAR $50+$ SCII; Group IV, NAR $100+$ SCII).

\section{Evaluating neurological and locomotor function}

Hind limb movement and neurological function were assessed on days 1, 3, 5, and 7 after SCII using the Basso, Beattie, and Bresnahan (BBB) motor rating scale. Twenty-one levels of the rating scale were used to evaluate BBB. A value of 0 indicated no detectable hind limb movement, 
$1-8$ indicated slight movement of the hind limb joints, 9 meant dorsal stepping, $10-20$ indicated progressively improved walking ability, and 21 meant normal movement without any discomfort.

\section{Assessment of edema}

Edema was measured according to the method described by Mdzinarishvili [18] with slight modifications. The dry and wet weights of spinal samples were quantified to calculate edema based on the percentage difference between the two weights (dry/wet weight).

\section{Evaluation of antioxidant activity}

SOD, GSH, and CAT activities and lipid peroxidation products (MDA) were evaluated in spinal tissue homogenates using commercial kits according to the experimental protocol.

\section{MPO activity}

Neutrophil infiltration was assessed by measuring MPO activity in spinal homogenates using an MPO assay kit according to the manufacturer's instructions. One unit of MPO activity was defined as the amount of enzyme required to degrade $1 \mathrm{mmol}$ peroxidase/min at 25 ${ }^{\circ} \mathrm{C}$ and is expressed as units per gram $(\mathrm{U} / \mathrm{g})$ of wet weight tissue.

\section{Inflammatory markers}

To analyze nuclear factor-kappa B (NF-kB) active subunit (NF-kB p65) in spinal homogenates, nuclear fractions were extracted using a nuclear extraction kit (Abcam, Cambridge, UK), and then levels were measured using an NF-KB p65 transcription factor assay kit (Abcam) according to the manufacturer's instructions. The levels of tumor necrosis factor- $\alpha$ (TNF- $\alpha$ ), IL- $1 \beta$, and IL- 6 were measured in spinal tissue cytosolic fractions using commercial ELISA kits (Thermo Fisher Scientific, Waltham, MA, USA) in accordance with the manufacturer's protocols.

\section{Western blot}

The protein content of samples was estimated using BCA assay kits (Beyotime, Biotechnology, Jiangsu, China). Equal protein quantities (40 $\mu \mathrm{g} / \mathrm{lane}$ ) were loaded into $8 \%$ sodium dodecyl sulfate-polyacrylamide gels and then transferred to polyvinylidene fluoride (PVDF) membranes. The membranes were blocked with $5 \%$ skimmed milk and Tween 20 containing Tris-buffered saline (TBS) and then incubated with primary antibodies overnight at $4{ }^{\circ} \mathrm{C}$. The following primary antibodies were used: rabbit polyclonal anti-NF p65 (1:1200; Santa Cruz Biotechnology, Santa Cruz, CA, USA), anti-TNF- $\alpha$ (1:2000; Santa Cruz), mouse anti-rat $\beta$-actin (1:500; Zhongshan Biotechnology, Beijing, China), and anti-rabbit-histone H3 (1:1000; Zhongshan Biotechnology; internal control). After TBS was used to remove unbound antibodies, membranes were incubated with anti-goat and anti-mouse secondary antibodies conjugated to horseradish peroxidase (HRP) (1:1000 and 1:2000, respectively; Santa Cruz Biotechnology) in TBS at room temperature for $1 \mathrm{~h}$. After washing again with TBS, the absorbance was assessed using an enhanced chemiluminescent system (Thermo Fisher Scientific, Waltham, MA, USA), and the signals were quantified using ImageJ software ( $\mathrm{NIH}$, Bethesda, MD, USA).

\section{Statistical analysis}

Data are expressed as mean \pm standard deviations (SDs). Significance differences between the control group and treatment groups were assessed using one-way analysis of variance (ANOVA) with Statistical Package for the Social Science (SPSS) software (IBM, New York, NY, USA). The least significant difference (LSD) was determined using post hoc multiplecomparison tests. $P<0.05$ was considered to indicate statistical significance.

\section{RESULTS}

\section{Effect of NAR on locomotor function}

Table 1 shows the locomotor function (hind limb movement or neurological function) of experimental rats. Sham-operated control rats exhibited normal hind limb movement on days 3 , 5 , and 7 . SCII rats exhibited significantly reduced $(p<0.01)$ hind limb movement, as evidenced by paraplegia and the lowest BBB score on days 1 , 3,5 , and 7 . However, treatment with NAR (50 and $100 \mathrm{mg} / \mathrm{kg})$ significantly improved $(p<0.05)$ the locomotor function by abolishing neuronal damage, as indicated by an improved BBB score compared with SCII rats.

\section{Effect of NAR on spinal edema}

The effects of NAR on spinal edema in experimental rats are illustrated in Fig. 1A. Although no edematic changes were observed in sham-operated rats, a considerable increase in the levels of spinal edema was seen in SCll rats $(P<0.01)$. NAR administration (500 and 100 $\mathrm{mg} / \mathrm{kg}$ ) significantly suppressed edema compared with SCII rats $(P<0.05)$. 
Table 1: Effect of NAR on spinal function (BBB score) in experimental rats

\begin{tabular}{lcccc}
\hline Group & $\mathbf{1}^{\text {st }}$ day & $\mathbf{3}^{\text {rd }}$ day & $\mathbf{5}^{\text {th }}$ day & $\mathbf{7}^{\text {th }}$ day \\
\hline Control & $19.00 \pm 2.00$ & $20.50 \pm 1.50$ & $21.00 \pm 2.00$ & $21.00 \pm 2.00$ \\
SCII & $4.01 \pm 0.3 \mathrm{a}^{\#}$ & $8.86 \pm 0.73 \mathrm{a}^{\#}$ & $11.85 \pm 1.25 \mathrm{a}^{\#}$ & $14.07 \pm 1.60 \mathrm{a}^{\#}$ \\
NAR 50 + SCII & $8.67 \pm 0.62 \mathrm{~b}^{*}$ & $13.81 \pm 1.20 \mathrm{~b}^{*}$ & $15.32 \pm 0.92 \mathrm{~b}^{*}$ & $16.64 \pm 1.80 \mathrm{~b}^{*}$ \\
NAR 100 + SCII & $9.44 \pm 0.91 \mathrm{~b}^{*}$ & $14.56 \pm 1.68 \mathrm{~b}^{*}$ & $16.76 \pm 0.78 \mathrm{~b}^{*}$ & $18.95 \pm 2.08 \mathrm{~b}^{\#}$ \\
\hline
\end{tabular}

Data are expressed as mean \pm SDs of 10 rats in each group; ${ }^{*} p<0.05,{ }^{\#} p<0.01$ SCIl group vs. sham control group (a) and treatment groups (NAR 50, 100) vs. SCIl group (b)

\section{Effect of NAR on MPO activity}

The effects of NAR on spinal MPO activity (neutrophil infiltration) in the experimental rats are shown in Fig. 1B). Spinal MPO activity was increased significantly $(P<0.01)$ in the SCII group compared with the sham-operated control group. Pre-treatment with NAR (50 and 100 $\mathrm{mg} / \mathrm{kg}$ ) for 7 days significantly reduced MPO activity $(P<0.01)$ via its anti-inflammatory effects.

\section{Effect of NAR on antioxidant activity}

The effects of NAR on the activity of endogenous antioxidant products in experimental rats are shown in Table 2. The activity of SOD, GSH, and CAT was significantly reduced in SCII rats compared with sham-operated control rats $(p<$ 0.05 ). Treatment with both $50 \mathrm{mg} / \mathrm{kg}$ and 100 $\mathrm{mg} / \mathrm{kg}$ NAR significantly increased the activity of SOD, GSH, and CAT $(p<0.05$ and $p<0.01$, respectively) compared with SCII rats by alleviating oxidative stress. Although the levels of lipid peroxidation products such as MDA were increased in the SCII group, treatment with NAR significantly lowered lipid peroxidation products (50 mg/kg, $p<0.01$; and $100 \mathrm{mg} / \mathrm{kg}, p<0.01$ ), confirming its antioxidant activity.
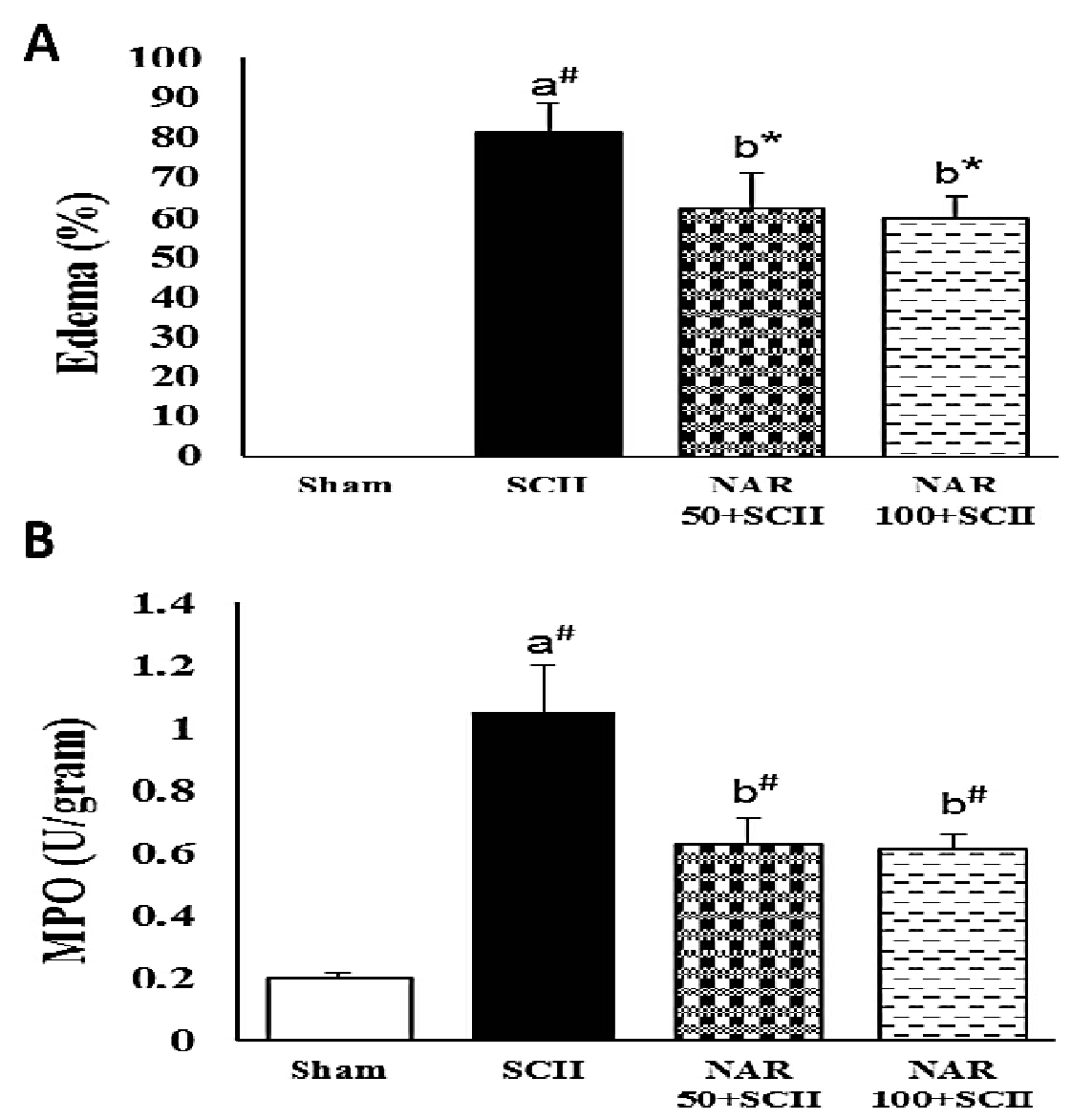

Figure 1: Effect of NAR on spinal edema $(A)$ and MPO activity $(B)$ in experimental rats. Data are expressed as mean \pm SD of 10 rats in each group; ${ }^{*} p<0.05,{ }^{\#} p<0.01$; SCll group vs. control group (a) and treatment groups (NAR 50, $100 \mathrm{mg} / \mathrm{kg}$ ) vs. SCll group 
Table 2: Effects of NAR on spinal lipid peroxidation and antioxidant activity in experimental rats

\begin{tabular}{lcccc}
\hline Group & $\begin{array}{c}\text { MDA (nmol/ } \\
\text { mg protein) }\end{array}$ & $\begin{array}{c}\text { SOD } \\
\text { (U/mg protein) }\end{array}$ & $\begin{array}{c}\text { CAT } \\
\text { (U/mg protein) }\end{array}$ & $\begin{array}{c}\text { GSH } \\
\text { ( } \boldsymbol{\mu g} / \mathbf{m g} \text { protein) }\end{array}$ \\
\hline Control & $0.82 \pm 0.09$ & $3.45 \pm 0.32$ & $66.60 \pm 7.53$ & $9.34 \pm 1.10$ \\
SCII & $1.65 \pm 0.12 \mathrm{a}^{\#}$ & $2.08 \pm 0.21 \mathrm{a}^{\#}$ & $48.04 \pm 4.09 \mathrm{a}^{\#}$ & $6.24 \pm 0.81 \mathrm{a}^{\#}$ \\
NAR 50 + SCII & $1.18 \pm 0.08 \mathrm{~b}^{*}$ & $2.99 \pm 0.38 \mathrm{~b}^{*}$ & $55.83 \pm 6.22 \mathrm{~b}^{*}$ & $7.84 \pm 0.77 \mathrm{~b}^{*}$ \\
NAR 100 + SCII & $0.92 \pm 0.11 \mathrm{~b}^{\#}$ & $3.24 \pm 0.46 \mathrm{~b}^{\#}$ & $61.72 \pm 9.14 \mathrm{~b}^{*}$ & $9.06 \pm 1.12 \mathrm{~b}^{\#}$ \\
\hline
\end{tabular}

Data are expressed as the means \pm SDs of 10 rats per group. ${ }^{*} p<0.05,{ }^{\#} p<0.01$; SCIl group vs. control group (a) and treatment groups (NAR 50,100 mg/kg) vs. SCIl group (b). One unit (U) of SOD inhibits the rate of increase in absorbance at $550 \mathrm{~nm}$ by $50 \%$ under assay condition. One unit (U) of CAT activity was defined as 1 $\mathrm{mg}$ of tissue proteins that consumed $1 \mu \mathrm{mol} \mathrm{H}_{2} \mathrm{O}_{2}$ at $405 \mathrm{~nm}$ in $1 \mathrm{~s}$.

Table 3: Effect of NAR on the activity of inflammatory markers in the spinal tissues of experimental rats

\begin{tabular}{lcccc}
\hline Group & $\begin{array}{c}\text { TNF-a } \\
\text { (pg/mg } \\
\text { protein) }\end{array}$ & $\begin{array}{c}\text { NF-p65 } \\
\text { (pg/mg } \\
\text { protein) }\end{array}$ & $\begin{array}{c}\text { IL-1 } \boldsymbol{~} \\
\text { (ng/mg } \\
\text { protein) }\end{array}$ & $\begin{array}{c}\text { IL-6 } \\
\text { (pg/mg } \\
\text { protein) }\end{array}$ \\
\hline Control & $135.72 \pm 14.50$ & $68.56 \pm 7.13$ & $73.06 \pm 8.22$ & $101.34 \pm 11.41$ \\
SCII & $310.02 \pm 26.77 \mathrm{a}^{\#}$ & $185.45 \pm 18.48 \mathrm{a}^{\#}$ & $194.50 \pm 17.25 \mathrm{a}^{\#}$ & $215.48 \pm 26.11 \mathrm{a}^{\#}$ \\
NAR 50 + SCII & $211.40 \pm 23.05 \mathrm{~b}^{\#}$ & $122.82 \pm 13.03 \mathrm{~b}^{\#}$ & $128.14 \pm 10.93 \mathrm{~b}^{*}$ & $157.11 \pm 15.09 \mathrm{~b}^{*}$ \\
NAR 100 + SCII & $154.83 \pm 16.49 \mathrm{~b}^{\#}$ & $81.08 \pm 9.62 \mathrm{~b}^{\#}$ & $90.13 \pm 11.01 \mathrm{~b}^{\#}$ & $120.11 \pm 13.68 \mathrm{~b}^{\#}$
\end{tabular}

Data are expressed as the means $\pm S D$ of 10 rats per group; ${ }^{*} p<0.05, p<0.01$; SCll group vs. control group (a) and treatment groups (NAR 50, $100 \mathrm{mg} / \mathrm{kg}$ ) vs. SCll group (b)

\section{Effects of NAR on inflammatory markers}

The effects of NAR on inflammatory markers including TNF- $\alpha$, NF-KB p65, IL-1 $\beta$, and IL-6 in experimental rats are presented in Table 3 . A significant increase in the levels of TNF- $\alpha, N F-k B$ p65, IL-1 1 , and IL- 6 was observed in the spinal tissue of $\mathrm{SCl}$ rats compared with sham-operated rats $(p<0.01)$. NAR administration significantly lowered the levels of TNF- $\alpha, N F-K B$ p65, IL-1 $\beta$, and IL-6, suggesting anti-inflammatory properties.

\section{Effects of NAR on protein expression}

Western blotting was employed to quantify the protein expression of inflammatory markers including TNF- $\alpha$ and NF-KB p65 in the spinal tissues of experimental rats Fig. 2. The expression of TNF- $\alpha$ (cytosolic fraction) and NFKB p65 (nuclear fraction) was significantly upregulated in the SCll group compared with the sham-operated control group $(P<0.01)$. Sevenday pretreatment with NAR significantly downregulated the protein expression of TNF- $\alpha$ and NF-kB p65.

\section{DISCUSSION}

The current study demonstrated that pretreatment with NAR for 7 days prior to SCII prevented neurological deficits and neuronal loss, and improved locomotor function. In addition, treatment with NAR substantially alleviated various pathological conditions such as edema, neutrophil infiltration (MPO), oxidative stress, and inflammation. NAR also downregulated the levels of major nuclear transcription factors (NF-kB p65) and proinflammatory cytokines (TNF- $\alpha$ ) to suppress the activation of glia and neutrophils (which produce excessive free radicals) and the subsequent inflammatory cascade. To the best of our knowledge, this is the first experimental study to demonstrate the neuroprotective effects of NAR against SCII.

The BBB screening scale is used to assess the neuroprotective efficacy of any drugs that are linked to hind limb movement [17]. The BBB score was significantly decreased in SCII rats because of oxidative stress (excessive free radical generation), which damages the myelin sheath of neurons, eventually leading to neuronal apoptosis or death. These results are consistent with a report by Fan et al [19]. Furthermore, NAR-treated rats had a significantly decreased BBB score, which was caused by NAR's free radical scavenging activity. Similarly, NAR supplementation after carotid artery occlusion (ischemia) substantially increased locomotor activity [14].

Spinal edema or water content was analyzed to check the integrity of the neurological system. During the induction of SCII, several vasoactive substances are synthesized that initiate the breakdown of the blood spinal cord barrier and increase blood pressure, leading to increased water content (edema) in the spinal cord [3]. 

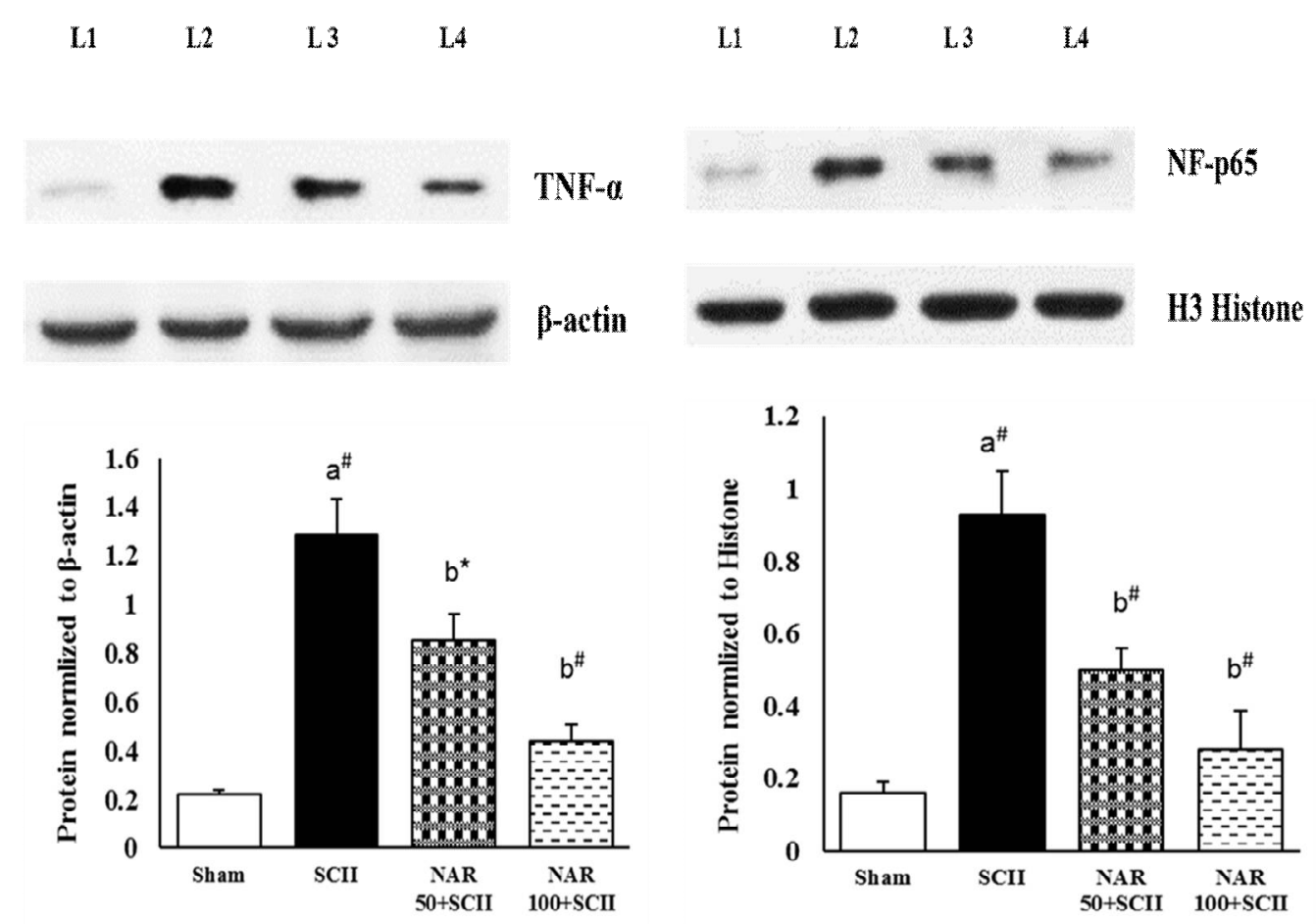

Figure 2: Effect of NAR on the protein expression of TNF- $\alpha$ (A) and NF-kB p65 (B) in the spinal homogenates of experimental rats. Values are expressed as the means \pm SDs of 10 rats in each group; ${ }^{*} p<0.05,{ }^{\#} p<0.01$; SCll group vs. control group (a) and treatment groups (NAR 50, $100 \mathrm{mg} / \mathrm{kg}$ ) vs. SCll group (b). Lane 1, sham control group; lane 2, SCll group; lane 3, NAR 50 group; lane 4, NAR 100 group. $\beta$-actin was used as the internal standard

Consistent with this, rats that underwent ischemia-reperfusion injury exhibited increased edema compared with the sham control group in the current study. However, treatment with NAR significantly lowered the levels of edema via its vasoprotective and neuroprotective activity. These results are consistent with the findings of Rong et al [8], who also indicated that NAR could reduce edema in a rat model.

The spinal tissue is extremely vulnerable to oxidative stress because it has a high oxygen utilization rate, high polyunsaturated fatty acids content, low levels of antioxidants, and high water content [20]. Several studies also suggested that ischemic-reperfusion injury reduced the activity of endogenous antioxidants, due to excessive free radical production (hypoxia), and subsequent oxidative stress $[21,22]$. Similarly, the activity of various endogenous antioxidant systems including SOD, GSH, and CAT was decreased, whereas MDA levels were increased due to the overproduction of free radicals (ROS/RNS) in the current study. Treatment with 50 and $100 \mathrm{mg} / \mathrm{kg}$ NAR improved antioxidant activity and reduced lipid peroxidation product formation. This suggests that NAR possesses potent free radical quenching ability and triggers endogenous antioxidant production. Moreover, previous studies revealed that NAR can activate nuclear factor-erythroid related factor-2, which is responsible for the synthesis of antioxidants, particularly SOD, CAT, and glutathione $[10,23]$.

Oxidative stress and inflammation play crucial roles in the pathogenesis of SCII. These two processes are interconnected (vicious cycle) and are thus implicated in most chronic diseases. After SCII insult, leukocytes (especially neutrophils and macrophages) begin to accumulate near the ischemic region and then slowly infiltrate into the spinal cord. They then activate microglial cells and other endothelial cells to stimulate the production of excessive amounts of inflammatory factors, including proinflammatory cytokines and free radicals [24]. The activated glial cells and neutrophils produce more inflammatory mediators, worsening the ischemic injury. Thus, leukocyte (neutrophil) infiltration inititates SCII. Accordingly, in the current study, the rate of neutrophil infiltration was assessed by measuring the activity of MPO in SCIl tissues. Spinal MPO activity was increased in the SCII group. However, treatment with NAR (50 and $100 \mathrm{mg} / \mathrm{kg}$ ) significantly reduced MPO activity via its anti-inflammatory activity. Golechha et al [25] demonstrated that different doses of NAR significantly lowered the 
levels of inflammatory mediators in a rat model via anti-inflammatory effects.

During SCII, activated glial cells and neutrophils might trigger an inflammatory cascade by upregulating NF-KB and its downstream genes, especially proinflammatory cytokines such as TNF- $\alpha, \quad$ IL-1 $\beta$, and IL-6. Therefore, the concentrations of inflammatory markers in spinal tissue were examined in the current study. Significant increases in the concentrations of TNF- $\alpha, \quad N F-k B$ p65, IL-1 $\beta$, and IL-6 were observed in the SCll group. However, NAR significantly reduced inflammatory marker concentrations to near-normal levels, thus confirming its anti-inflammatory activity. These data are consistent with the observations by Chen et al [26], who demonstrated that NAR could lower the levels of lower inflammatory markers.

$\mathrm{NF}-\mathrm{KB}$ is a nuclear transcriptional factor that is highly expressed in infected tissues or cells. It modulates the expression of many inflammatory markers and proinflammatory cytokines and thereby regulates the inflammatory cascade [26]. The abovementioned biochemical analyses revealed that NAR attenuates the concentration of various inflammatory markers; to confirm these results, the protein expression of TNF- $\alpha$ and NF-KB p65 were quantified. Previous studies have revealed that the activation of NF-KB p65 is a crucial event during the induction of SCII in several animal models [3,27]. As indicated above, the protein expression of both TNF- $\alpha$ (cytosolic fraction) and NF-kB p65 (nuclear fraction) was significantly upregulated during SCII due to excessive oxidative stress and inflammation. Pretreatment with NAR significantly downregulated the protein expression of TNF- $\alpha$ and NF-KB p65, indicating its antioxidant and anti-inflammatory activities. Sahu et al reported that supplementation with NAR significantly downregulated the protein expression of NF-KB and thus decreased proinflammatory cytokine production in a rat model [13]. Hence, we propose that NAR directly inhibits the activation of NF-KB, especially in nuclear fractions, and thus suppresses the production of pro-inflammatory cytokines.

\section{CONCLUSION}

Pretreatment with 50 and $100 \mathrm{mg} / \mathrm{kg}$ NAR afford effective neurotherapeutic activity by increasing antioxidant activity to restrain oxidative stress. NAR supplementation also inhibits excessive inflammatory cytokine production by inhibiting the activation of NF-KB p65 and TNF- $\alpha$ in a rat model of SCII. Thus, NAR enhances locomotor activity and protects spinal tissue from SCII insultinduced damage. Both doses of NAR also stimulate potent neuroprotective activity, although $100 \mathrm{mg} / \mathrm{kg}$ of NAR-100 was more potent than the $50 \mathrm{mg} / \mathrm{kg}$ dose. Future studies are required to confirm these findings as well as elucidate the mechanism of these neuroprotective effects.

\section{DECLARATIONS}

\section{Acknowledgement}

Financial aid for this work was received from Ankang City Central Hospital, Ankang, Shanxi 725000, China.

\section{Conflict of Interest}

No conflict of interest associated with this work.

\section{Contribution of Authors}

The authors declare that this work was done by the authors named in this article and all liabilities pertaining to claims relating to the content of this article will be borne by them.

\section{Open Access}

This is an Open Access article that uses a funding model which does not charge readers or their institutions for access and distributed under the terms of the Creative Commons Attribution License (http://creativecommons.org/licenses/by 14.0) and the Budapest Open Access Initiative (http://www.budapestopenaccessinitiative.org/rea d), which permit unrestricted use, distribution, and reproduction in any medium, provided the original work is properly credited.

\section{REFERENCES}

1. Saxena P, Abu Hasan F, Merry C. Spinal cord ischemia following coronary artery bypass surgery. $J$ Card Surg 2012; 27(1): 45-46.

2. Liu JJ, Wang XW, Wu SF, Wu QN, Hao DJ. Salvia miltiorrhiza aqueous root extract plays an important role in improving locomotor activity in rats with spinal cord injury. Trop J Pharm Res 2016; 15(8): 1667-1672.

3. Ning $N$, Dang $X$, Bai C, Zhang C, Wang K. Panax notoginsenoside produces neuroprotective effects in rat model of acute spinal cord ischemia-reperfusion injury. $J$ Ethnopharmacol 2012; 139(2): 504-512.

4. Schallert T, Fleming SM, Leasure JL, Tillerson JL, Bland ST. CNS plasticity and assessment of forelimb sensorimotor outcome in unilateral rat models of stroke, 
cortical ablation, parkinsonism and spinal cord injury. Neuropharmacol 2000; 39(5): 777-787.

5. Dumont RJ, Okonkwo DO, Verma S, Hurlbert RJ, Boulos $P T$, Ellegala $D B$, Dumont AS. Acute spinal cord injury, part l: pathophysiologic mechanisms. Clin Neuropharmacol 2001; 24(5): 254-264.

6. Kam A, Li KM, Razmovski-Naumovski V, Nammi S, Chan $K, \mathrm{Li} Y, Q \mathrm{Li} G$. The protective effects of natural products on blood-brain barrier breakdown. Curr Med Chem 2012; 19(12): 1830-1845.

7. Khalatbary AR. Natural polyphenols and spinal cord injury. Iran Biomed J 2014; 18(3): 120-129.

8. Rong W, Wang J, LiU X, Jiang L, Wei F, Hu X, Han X, Liu $Z$. Naringin treatment improves functional recovery by increasing BDNF and VEGF expression, inhibiting neuronal apoptosis after spinal cord injury. Neurochem Res 2012; 37(8): 1615-1623.

9. Bharti S, Rani N, Krishnamurthy B, Arya DS. Preclinical evidence for the pharmacological actions of naringin: a review. Planta Medica 2014; 80(06): 437-451.

10. Gopinath K, Sudhandiran G. Naringin modulates oxidative stress and inflammation in 3-nitropropionic acid-induced neurodegeneration through the activation of nuclear factor-erythroid 2-related factor-2 signalling pathway. Neurosci 2012; 227: 134-143.

11. Benavente-Garcia O, Castillo J. Update on uses and properties of citrus flavonoids: new findings in anticancer, cardiovascular, and anti-inflammatory activity. J Agri Food Chem 2008; 56(15): 6185-6205.

12. Choe SC, Kim HS, Jeong TS, Bok SH, Park YB. Naringin has an antiatherogenic effect with the inhibition of intercellular adhesion molecule-1 in hypercholesterolemic rabbits. J Cardiovasc Pharmacol 2001; 38(6): 947-955.

13. Sahu BD, Tatireddy S, Koneru M, Borkar RM, Kumar JM, Kuncha $M$, Srinivas $R$, Sistla $R$. Naringin ameliorates gentamicin-induced nephrotoxicity and associated mitochondrial dysfunction, apoptosis and inflammation in rats: possible mechanism of nephroprotection. Toxicol Appl Pharmacol 2014; 277(1): 8-20.

14. Gaur V, Aggarwal A, Kumar A. Protective effect of naringin against ischemic reperfusion cerebral injury: possible neurobehavioral, biochemical and cellular alterations in rat brain. Eur J Pharmacol 2009; 616(1): 147-154.

15. Singh $D$, Chopra $K$. The effect of naringin, a bioflavonoid on ischemia-reperfusion induced renal injury in rats. Pharmacol Res 2004; 50(2): 187-193.

16. Rani N, Bharti S, Manchanda M, Nag TC, Ray R, Chauhan SS, Kumari S, Arya DS. Regulation of heat shock proteins 27 and 70, p-Akt/p-eNOS and MAPKs by Naringin Dampens myocardial injury and dysfunction in vivo after ischemia/reperfusion. PLoS One 2013; 8(12): e82577.

17. Hwang JY, Min SW, Jeon YT, Hwang JW, Park SH, Kim $J H$, Han SH: Effect of coenzyme Q10 on spinal cord ischemia-reperfusion injury. J Neurosurg Spine 2015; 22: 432-438.

18. Mdzinarishvili A, Kiewert C, Kumar V, Hillert M, Klein J. Bilobalide prevents ischemia-induced edema formation in vitro and in vivo. Neurosci 2007; 144: 217-222.

19. Fan ZK, Wang YF, Cao Y, Zhang MC, Zhang Z, Lv G, Lu $W$, Zhang $Y Q$. The effect of aminoguanidine on compression spinal cord injury in rats. Brain Res 2010; 1342: 1-10.

20. Xiong $Y$, Rabchevsky AG, Hall ED. Role of peroxynitrite in secondary oxidative damage after spinal cord injury. $J$ Neurochem 2007; 100(3): 639-649.

21. Zhai $Q$. The neuro-protective effects of water extract of Faces Trogopterori on transient MCAO rats. Biomed Res 2016; 27(3): 972-976.

22. Chen F, Zhang N, Ma X, Huang T, Shao Y, Wu C, Wang Q. Naringin Alleviates Diabetic Kidney Disease through Inhibiting Oxidative Stress and Inflammatory Reaction. PLoS One 2015; 10(11): e0143868.

23. Reuter S, Gupta SC, Chaturvedi MM, Aggarwal BB. Oxidative stress, inflammation, and cancer: how are they linked? Free Radic Biol Med 2010; 49(11): 16031616.

24. Fleming JC, Norenberg $M D$, Ramsay DA, Dekaban GA, Marcillo $A E$, Saenz AD, Pasquale-Styles M, Dietrich $W D$, Weaver $L C$. The cellular inflammatory response in human spinal cords after injury. Brain 2006; 129(12): 3249-3269.

25. Golechha M, Chaudhry U, Bhatia J, Saluja D, Arya DS. Naringin protects against kainic acid-induced status epilepticus in rats: evidence for an antioxidant, antiinflammatory and neuroprotective intervention. Biol Pharm Bull 2011; 34(3): 360-365.

26. Chen L, Guo WC. Dipsacus asperoides (Xue Duan) inhibits spinal cord injury-induced inflammatory responses in rats. Trop J Pharm Res 2016; 15(4): 729733.

27. Smith $P D$, Puskas $F$, Meng $X$, Lee $J H$, Cleveland JC Jr, Weyant MJ, Fullerton DA, Reece TB. The evolution of chemokine release supports a bimodal mechanism of spinal cord ischemia and reperfusion injury. Circulation 2012; 126: 110-117. 